\title{
Bistatic LIDAR experiment proposed for the shuttle/tethered satellite system missions
}

\author{
David J. McComas \\ Los Alamos National Laboratory, Los Alamos, New Mexico 87545 \\ Harlan E. Spence \\ University of California, Los Angeles, California 90024 \\ Robert R. Karl and Henry G. Horak \\ Los Alamos National Laboratory, Los Alamos, New Mexico 87545
}

Thomas D. Wilkerson

University of Maryland, College Park, Maryland 20742

(Received 5 November 1984; accepted for publication 17 January 1985)

\begin{abstract}
A new experiment concept has been proposed for the shuttle/tethered satellite system missions, which can provide high-resolution, global density mappings of certain ionospheric species. The technique utilizes bistatic LIDAR to take advantage of the unique dual platform configuration offered by these missions. A tuned, shuttle-based laser is used to excite a column of the atmosphere adjacent to the tethered satellite, while triangulating photometric detectors on the satellite are employed to measure the fluorescence from sections of the column. The fluorescent intensity at the detectors is increased about six decades over both ground-based and monostatic shuttle-based LIDAR sounding of the same region. In addition, the orbital motion of the shuttle provides for quasiglobal mapping unattainable with ground-based observations. Since this technique provides such vastly improved resolution on a synoptic scale, many important middle atmospheric studies, heretofore untenable, may soon be addressed.
\end{abstract}

\section{INTRODUCTION}

Remote LIDAR measurements of ionospheric species have been successfully made from the ground and from aircraft. The technique involving resonance scattering was first carried out for sodium in the $90-\mathrm{km}$ layer shortly after the development of the tunable dye laser. 'With improvements in laser/LIDAR technology, the ground-based observations were extended to other species such as potassium ${ }^{2}$ and to phenomena such as wave structures in the upper atmosphere. $^{3}$ The recent University of Illinois/Goddard SFC work ${ }^{4}$ demonstrates that these observations can also be made from aircraft and opens the way towards a general class of ionospheric LIDAR measurements that can be made from moving platforms. In the limit of orbiting platforms, such as the shuttle, this affords the attractive prospect of global coverage and a much more comprehensive view of the behavior of many physical processes in the ionosphere. NASA's shuttle LIDAR report ${ }^{5}$ examines many such studies and features LIDAR profiling of sodium as one of the early, desirable targets.

During the late 1980's, NASA will fly a series of missions involving the space shuttle with a tethered satellite system (TSS) ${ }^{6}$; thereby creating dual-platform observing configurations with the tethered satellite (TS) and the shuttle. A new experiment concept, suggested by McComas and Spence, ${ }^{7}$ which takes advantage of this unique configuration, has recently been proposed ${ }^{8}$ in response to NASA's TSS announcement of opportunity. The experiment, if accepted, will provide global high-resolution density profiles of certain ionospheric species. The technique, laser induced fluorescence (LIF), utilizes a bistatic LIDAR configuration to provide triangulated photometric observations from the tethered satellite of a fluorescent column excited by a laser on the shuttle. Figure 1 illustrates the LIF experiment configuration. This method has advantages over both groundbased monostatic LIDAR and over shuttle-based, downward looking LIDAR, the major advantage being that the excited region under study is much closer to the detectors, giving a vastly improved signal-to-noise ratio. During TSS "tether-down" missions, the high-resolution global density profiles obtained by the LIF technique will be extremely valuable for the development of more detailed understanding of middle atmospheric physics.

\section{THE EXPERIMENTAL CONCEPT}

The TS system in the tether-down mode places the shuttle at $\sim 200-\mathrm{km}$ altitude with the TS in the $\sim 100$ to $150-\mathrm{km}$ altitude regime. This region has not been experimentally sampled in detail, since it is too high for balloons and too low for long-lived satellites. Currently our knowledge about this region has been acquired from limited and often ambiguous observations made by short duration sounding rocket traversals, ground-based remote sensors (airglow photometry and LIDAR), and from the results of ionospheric modeling. The LIF technique, invented to take full advantage of the unique dual platform configuration offered by the TSS mis. sions, is capable of providing data with accuracies heretofore unattainable, in this altitude region. By this method, high precision, global density measurements of various ionic and neutral species can be made. Shuttle-based lasers can be tuned to a particular resonant frequency to excite specific 


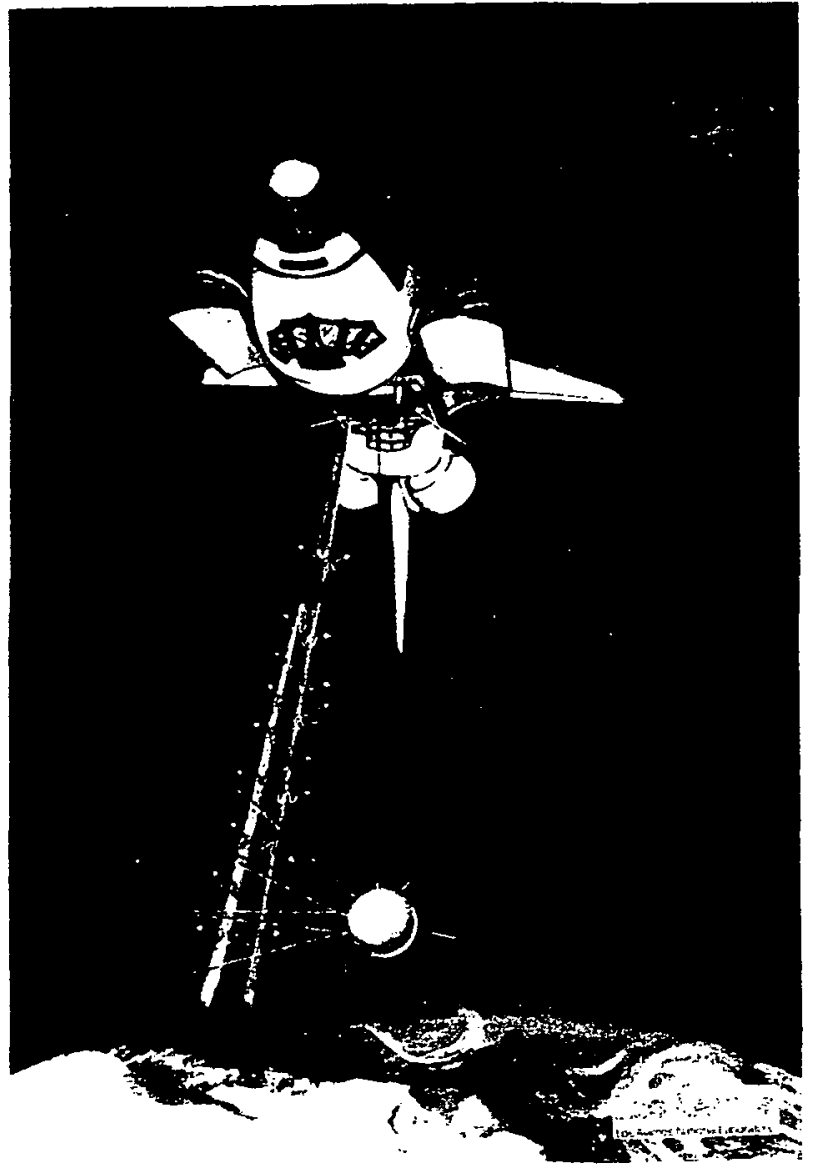

FIG. 1. The LIF experiment configuration on a tether-down shuttle/tethered satellite system mission. A tunable laser on the shuttle excites a column of the atmosphere adjacent to the tethered satellite. Fluorescence from various sections of the column is measured with photometers situated on the nearby tethered satellite.

species within a column of the ionosphere adjacent to the TS. Induced fluorescence from different altitude bands along this column is then observed on the TS by an array of photometers sensitive to the fluorescence. By accurately knowing the location of the TS with respect to the excitation beam, the returned signals can be used to derive concentration profiles.

While the LIF experiment configuration essentially constitutes a bistatic LIDAR, it has numerous advantages over ground-based and shuttle-only-based monostatic LIDAR. The configuration of the TSS missions allows detectors to be located very close to the region that is being probed. The irradiance seen at a detector drops off approximately as the inverse square of the distance from the fluorescent region, and the proximity of thie excited region to the detector is, therefore, a major factor in the signal magnitude observed at the detector. For the LIF configuration the path length between the excited region and the detector is $\sim 100$ $m$, while the path length for either ground- or shuttle-based LIDAR is $\sim 100 \mathrm{~km}$; the intensity at the detector is, therefore, $\sim 10^{6}$ times greater. Ground-based LIDAR has the additional problem that optical paths must extend through the troposphere where substantial extinction occurs at many wavelengths. Similarly, in the sun-lit hemisphere, shuttlebased monostatic LIDAR must measure backscattered re- turns from these regions against the bright tropospheric background. In contrast to these techniques, the LIF method guarantees the observation of nearby regions as well as excluding optical paths that include high background and extinction regions.

\section{INSTRUMENTATION}

The LIF technique may be applied to many ionospheric species, both neutral and ionic. The applicability of the technique to a wide variety of investigations necessarily precludes a single description for all possible LIF instrumentation and hardware. Instead, a general description of the technique is more appropriate to encompass presently envisioned LIF experiments. The LIF experiment configuration (see Fig. 1) would utilize a tunable pulsed laser onboard the shuttle to excite fluorescence of an atmospheric species along the conical laser-beam path directed to a position $\sim 100 \mathrm{~m}$ from the TS. The choice of laser would depend primarily upon the trace species to be investigated, the resonant and fluorescent wavelengths that are available for that species, and the ability of a laser to emit at one of these wavelengths.

By choosing to illuminate a region near the TS-mounted detectors, as opposed to relying upon long path length backscatter, the very large return signal strength provided by this experiment configuration makes it feasible to utilize numerous wavelengths with smaller absorption cross sections. While present laser technology makes it impossible to access many of the large absorption cross-section wavelengths, conventional lasers are suitable to access some with smaller cross sections. Consequently, certain species inaccessible to present LIDAR experiments may now be observed. In addition, it is possible to use lower power lasers than are required for monostatic shuttle-based LIDAR. The advantages of this are multifold, namely longer laser lifetime, narrower linewidths, easier tunability, smaller size, lower power requirements, easier production of a high repetition rate, and lower cost.

Regardless of species choice, a precision spectrometer, or the optogalvanic effect ${ }^{9}$ in a hollow cathode lamp, will be used to automatically maintain overlap of the laser wavelength with the chosen fluorescent line. Wavelength tuning of the laser line (accurate to $10 \%-20 \%$ of the laser linewidth) will correct for the Doppler shift of the line due to the orbital motion of the shuttle. The perceived shift is proportional to the orbital velocity $(\sim 7 \mathrm{~km} / \mathrm{s})$ over the speed of light, times the sine of the small angle between the shuttle TS separation vector and nadir. For the maximum envisioned angle of $\sim 5^{\circ}$, the shift amounts to $\sim \pm 0.012 \AA$, which is of the order of visual, ionospheric Doppler linewidths.

An array of photometers, mounted at various polar angles on the TS, will sample the signal returned from the nearby fluorescent region. These photometers will have aperture areas $-10 \mathrm{~cm}^{2}$, focal lengths of $5-8 \mathrm{~cm}(\sim f / 2)$, and a variety of fields of view $\left(\sim 7 \times 10^{2} \mathrm{sr}\right)$. Each photomultiplier detector will have an interference filter wheel to allow for selective observation at specific wavelengths. With this array induced fluorescence will be measurable, as well as passive photometry of natural airglow and TS-induced heating emissions. 


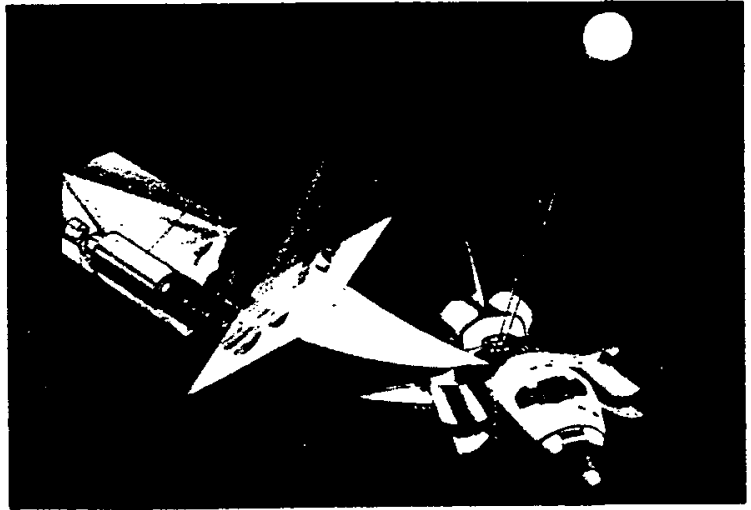

FIG. 2. The laser directional ranger (LDR) subsystem provides the precise tethered satellite locational information required to point the LIF excitation laser and reduce the photometer data.

An additional and important supporting component of this system is a subsystem for locating the TS and for differential pointing of the LIF laser. This laser directional ranger (LDR) subsystem provides a means for the accurate location and ranging of the TS with respect to the shuttle. Such a system not only assures the proper coordinated pointing required, but also ultimately yields the fluorescent region to TS separation necessary for the conversion of LIF measured signals to actual species densities. The basic LDR technique calls for the timing of a laser beam directionally pulsed from the shuttle to the TS and reflected back. By utilizing modern laser and optical techniques, the TS can be located to within $\sim 10 \mathrm{~m}$ horizontally at a $100-\mathrm{km}$ distance from the shuttle, while the range is found to within only a few centimeters. The LDR subsystem (see Fig. 2) consists of five integrated components; corner-cube reflector, laser source, photometric detector, tracking mirror system, and instrument electronics and microprocessor.

A passive lightweight corner-cube reflector mounted on the shuttle side of the TS will perform the requisite reflection of the laser pulses. Corner-cube reflectors have the essential property that any reflected beam path is always parallel to the incident beam path. On the shuttle, a $1.06-\mu \mathrm{m}$ neodymium YAG laser capable of delivering $\sim 2 \mathrm{~mJ} /$ pulse at repetition rates up to $\sim 100 \mathrm{~Hz}$, will supply the necessary pulsed beam source. Analysis indicates that a pulse duration of $\sim 10 \mathrm{~ns}$ and a beam divergence angle of $\sim 1 \mathrm{mrad}$ will provide ample signal over background levels for this application and will yield a beam well within ANSI eye safety criteria for ground-based observers.

The reflected beam detecting photometer, also on the shuttle, will consist of a $100-\mathrm{cm}^{2}$ aperture telescope and a silicon avalanche quadrant detector with an $\sim 10-\AA$ passband filter at $1.06 \mu \mathrm{m}$. The field of view will be $1-4 \mathrm{mrad}$ and the system focal length $\sim 25 \mathrm{~cm}(\sim f / 2.2)$ for a quadrant detector chip diameter of $\sim 0.1 \mathrm{~cm}$. Using this detected signal, the reflected image in the system field of view can be automatically adjusted with a tracking mirror system to continuously determine the TS location and precisely point the laser/detector mirror assembly along a parallel axis. The mirror pointing system consists of a mirror, optically flat to $\sim \frac{1}{2}$ wavelength, which can be pointed repeatedly to within $0.1 \mathrm{mrad}$ and incrementally by $0.03 \mathrm{mrad}$.

Associated logic and electronics will control the coordination of the shuttle-based LDR components. In particular, the quadrant detector output will be used to continuously determine pointing mirror motions. Tracking of the TS is thereby maintained throughout the mission. During initial acquisition, and in the event that the TS location is lost, a search mode will automatically be initiated by the internal logic. The internal logic will also determine the range, from source/detector timing, and format the range and direction angle data for telemetry down link. Additional logic will be responsible for laser firing, photometer data recording, pointing angle, wavelength control, system check and activation, and operation control of the entire LIF system.

\section{FEASIBILITY STUDY}

The LIF experiment technique can be applied to the investigation of numerous species. Atomic and molecular species of interest for middle atmospheric research include $\mathrm{O}, \mathrm{NO}, \mathrm{Mg}, \mathrm{Ca}, \mathrm{Fe}$ (as well as their singly ionized states), in addition to $\mathrm{O}_{2}^{+}, \mathrm{OH}, \mathrm{NO}_{2}, \mathrm{~N}_{2}^{+}, \mathrm{Na}, \mathrm{K}$, and $\mathrm{LI}$. Based on criteria discussed previously for laser selection, species number density at the altitude of study, and optical properties of the target species, some of the species may be studied with existing lasers, while others require further laser development.

$\mathrm{Na}, \mathrm{K}$, and NO all appear to be excellent candidates for LIF experimentation on early tether-down TSS missions. This choice is based primarily upon both high scientific yield and the presently available laser technology required. Studies conducted at altitudes near $100 \mathrm{~km}$ will place the LIF photometers near the peaks of the sodium and potassium layers. Conveniently, sodium has a readily accessible resonant line at $589 \mathrm{~nm}$ (as does potassium at $770 \mathrm{~nm}$ ) and is a good species for investigations of ionospheric chemistry and transport and the propagation of waves and tides. This section then, as an example of the general feasibility of LIF experimentation, addresses the feasibility of observing sodium (Na-LIF) near $100 \mathrm{~km}$. The results for potassium are similar to those derived here for sodium.

There are two promising approaches to obtain the laser line at $589 \mathrm{~nm}$ for NA. A tunable dye laser may be employed such as the NASA/CNRS laser system ${ }^{10}$ identified for the ER-2 DIAL program which already exceeds the laser energy requirements for the LIF configuration. The second approach yields $50-100 \mathrm{~mJ}$ at $589 \mathrm{~nm}$ by a two-wave mixing process in a nonlinear crystal, by combining a slightly tunable YAG laser at $1.06 \mu \mathrm{m}$ with a YAG laser at $1.32 \mu \mathrm{m}$. The desired wavelength is obtained within only a 0.01 to $0.02-\AA$ linewidth. ${ }^{11}$

In order to assess the feasibility of the $\mathrm{Na}$-LIF experiment, the signal-to-background ratio must be considered. For this calculation, the laser is assumed to have a character. istic energy of $50 \mathrm{~mJ} /$ pulse, pulse duration of $10 \mathrm{~ns}$, beam divergence angle of $0.1 \mathrm{mrad}$, and is directed down to $\sim 100$ $m$ from the TS. The TS-mounted photometer has an aperture of $10 \mathrm{~cm}^{2}$ and a field of view of $45^{\circ}$ vertical $\times 5^{\circ}$ horizontal. 
The laser pulse is resonantly scattered by sodium atoms present in successive volumes along a conical column, and some of this scattered light enters the field of view of the photometer. The LIDAR equation can be used to calculate such fluxes $F$ and gives $F \simeq(\operatorname{Ln} \sigma \Delta l) \omega / 4 \pi$, where $L$ is the laser energy $(J)$ per duration of the fluorescent signal observed by the photometer, $n$ is the concentration of $\mathrm{Na}$ $\left(\mathrm{cm}^{-3}\right), \sigma$ is the effective scattering cross section per particle $\left(\mathrm{cm}^{2}\right), \Delta l$ is the vertical length of the field of view, and $\omega$ is the solid angle of the photometer aperture as seen from the emitting volume. Using values of $n=2 \times 10^{3} \mathrm{~cm}^{-3}$ and $\sigma=6 \times 10^{-12} \mathrm{~cm}^{2}$ yields a flux of $\sim 0.2 \mu \mathrm{W}$.

A background in the sun-lit hemisphere exists due to the resonant scattering of the solar $\mathrm{Na}$-Fraunhofer light by the Na layer. The breadth at the bottom of this Fraunhofer line is large $(0.1 \AA)$ compared to the Doppler width $(0.016 \AA)$ of ionospheric $\mathrm{Na}$. The residual solar flux at the bottom of the line is $\sim 5 \%$ that of the solar continuum just outside the line. ${ }^{12}$ An estimate of the flux of sunlight resonantly scattered into the field of view can be obtained from single-scattering theory. A value of $\sim 0.8 \mathrm{nW}$ is obtained yielding a very favorable signal-to-background ratio of $\sim 250$ per shot. This ratio dramatically increases in the nighttime hemisphere where the scattered solar background constitutes no problem.

A similar signal-to-background calculation is made for the LDR subsystem. For the described system with laser energy of $2 \mathrm{~mJ} /$ pulse, pulse duration of $10 \mathrm{~ns}$, and a beam divergence of $1 \mathrm{mrad}$; a corner-cube reflector with a $5.08-\mathrm{cm}$ diameter; and a photometer with aperture area $(A)$ of 100 $\mathrm{cm}^{2}$ and field of view of $3 \mathrm{mrad}$, the return signal may be calculated. For a shuttle-TS separation of $100 \mathrm{~km}$ the laser flux intercepted by the reflector is $\sim 5.2 \times 10^{7} \mathrm{~mJ}$. This flux is returned, at the shuttle, in a diffraction pattern produced by the corner-cube reflector. The half-divergence angle of the pattern is $0.02 \mathrm{mrad}$ and the pattern diameter at the receiver is therefore $\sim 4.2 \mathrm{~m}$. The detector area intercepts $3.8 \times 10^{-10} \mathrm{~mJ}$ or $2.0 \times 10^{6}$ photons during a single $10-\mathrm{ns}$ pulse.

For a photometer looking down at the solar illuminated Earth, the background flux is given by the expression $F^{*}=A \Omega I^{*} \Delta \lambda$, where $\Omega$ is the photometer field of view, $I^{*}$ is the radiance of the Earth, and $\Delta \lambda$ the filter bandwidth. $I^{*}$ is calculated using Lambert's law of reflection $I^{*}=a H \cos \theta$ / $\pi$, where $a$ is the albedo of the reflecting surface, $H$ is the solar irradiance incident on the top of the atmosphere, and $\theta$ is the zenith angle of the sun at the reflecting area. Taking the solar irradiance on the atmosphere to be $6.5 \times 10^{-3} \mathrm{~mW} /$ $\mathrm{cm}^{2} \AA$ and using a 10- $\AA$ pass band filter and an albedo of unity, a maximum background flux of $\sim 800$ photons is obtained in $10 \mathrm{~ns}$. The resultant worst case signal-to-background ratio is $\sim 2500$ demonstrating that the LDR subsystem is quite feasible.

\section{DISCUSSION}

The LIF technique can provide a new method for ionospheric investigation, based on previously unattainable high resolution global density mappings. The flexibility of this technique to determine profiles of many trace species throughout the middle atmosphere makes this an attractive improvement over the present techniques available for probing this region. With higher-precision data gathered on a synoptic scale, an improved understanding of many ionospheric phenomena will be possible.

With this technique, several avenues of investigation are envisioned. An immediate result of LIF observations are global quasi-three-dimensional density distributions of various ionospheric species. These data are gathered over long length scales in relatively short time scales, thus facilitating the study of winds, tides, and atmospheric gravity waves. Fine structure and temporal and spatial variability of these data can yield information on diffusion, electromagnetic fields, and turbulence. Finally, the ability to probe day-night transitions and orbit relative to chemical releases will reveal basic chemical rate processes involving ionization, dissociation, and recombination.

\section{ACKNOWLEDGMENTS}

We gratefully acknowledge contributions to the development of this experiment from all of the other investigators on our NASA proposal: Melvin I. Buchwald, Marie-L. Chanin, Donald D. Cobb, Paul J. Coleman, Chester S. Gardner, Arieh Rosenberg, George L. Siscoe, and John Zinn. Work described herein was principally carried out under the auspices of the U. S. Department of Energy, Los Alamos National Laboratory; Institute for Geophysics and Planetary Physics, Los Alamos; University of California, Los Angeles; and the University of Maryland. One of the authors (H.S.) was supported by the Office of Naval Research.

'A. Gibson and M. Sanford, Nature 239, 509 (1971).

${ }^{2}$ G. Megie, F. Bos, J. Blamont, and M. Chanin, Planet. Space. Sci. 26, 27 (1978).

${ }^{3}$ J. Rowlett, C. Gardner, E. Richter, and C. Sechrist, Geophys. Rev. Lett. 5, 683 (1978).

${ }^{4} A$. Segal, D. Voelz, C. Gardner, and C. Sechrist, Conference Digest, Twelfth International LASER-RADAR Conference, Aix-en-Provence, France, 1984

sAtmospheric LIDAR Working Group, NASA Report No. SP-433, National Technical Information Service, Springfield, VA, 1979.

${ }^{6}$ NASA A.O. No. OSSA-1-84, 15 April 1984.

'D. McComas and H. Spence, Institute of Geophysics and Planetary Physics Report No. 2548, University of California at Los Angeles, Los Angeles. CA, 15 April 1984.

${ }^{8}$ D. Cobb, G. Siscoe, M. Buchwald, M. L. Chanin, P. Coleman, C. Gardner,-H. Horak, R. Karl, Jr.. D. McComas, A. Rosenberg, H. Spence, T. Wilkerson, and J. Zinn, Proposal to the National Aeronautics and Space Administration for a Bistatic LIDAR for Ionospheric Species Mapping and for a Precise Locating LIDAR for the Tethered Satellite System, 17 July 1984(Los Alamos National Laboratory, Los Alamos, NM, 1984).

${ }^{9}$ R. A. Keller, R. Engleman, and E. F. Zalenski, J. Opt. Soc. 69, 738 (1979).

${ }^{10}$ E. Browell, G. Megie, P. Flamont, W. Hall, M. Bourdet, and J. Talbot, Twelfth International Laser-Radar Conference, edited G. Megie (Service d'Aeronomie du CNRS, Verriers-b-Buisson. France, 1984), abstracts.

'J. Bufton, C. Gardner, and C. Philbrick, Proceedings of Optical Society, Conference on Optical Remote Sensing of the Atmosphere, 15-18 January 1985, Incline Village, NV, pp. WC29-1, WC29-5, 1985.

${ }^{12} \mathrm{C}$. Allen, in Astrophysical Quantities (University of London, London 1963). 